\title{
Breast cancer, chemotherapy and treatments
}

\author{
$\mathrm{Li}^{-P i n} \mathrm{Kao}^{1,2 *}$ \\ ${ }^{1}$ Department of Basic Medical Sciences, Purdue University, West Lafayette, Indiana, USA \\ ${ }^{2}$ Centre for Cancer Research, Purdue University, West Lafayette, Indiana, USA
}

\begin{abstract}
Breast cancer, the first most common malignant tumor in women worldwide. Many etiological factors such as a wide spectrum of clinical manifestations caused by family clustering, hormonal factors, physiology changed, environmental inducers, and life styles. Although there are many FDA-approval drugs for breast cancers. Tumors are heterogenous and individual has inherited different genetic background. Thus, it is hard to find an effective treatment and it may recurrent after recovery from first treatment.
\end{abstract}

\section{Introduction}

The mortality rate exceeds 144,000 cases and most of the breast cancer (BC) cases are resistant to traditional chemotherapy and radiotherapy. A wide variety of chemotherapeutic agents have been tried and are in use, including tamoxifen, docetaxel, anthracyclines (i.e. doxorubicin, and epirubicin), taxanes (i.e. paclitaxel, and docetaxel), 5 -fluorouracil, cyclophosphamide, and carboplatin. No regimen has been proved to be curative. Often, the response rate and prolongation of survival are minimal (a few months or less), and there is a significant morbidity associated with poor treatment effects. Surgical resection is considered the first-line options for early tumors, although there is no agreement on which is the best approach. Interestingly, triple negative has been reported has higher chemo-resistance than other type of breast cancers with low autophagy activity [1,2]. Antiestrogen resistant cell lines exhibit increased basal autophagy when compared with their antiestrogen sensitive parental cells [3]. Study has shown to manipulate transient receptor potential channel 5 (TRPC5), a Ca2+ permeable cation channel, which helps in promotes autophagic activity [4]. Studies also demonstrate the breast cancer usually prosurvival after autophagy activity increase by various therapeutics [5-7]. Moreover, Tamoxifen and Faslodex (ICI) both induce autophagy in ER+ breast cancer cells without $[3,5,8-11]$. There are small molecules which has been in FDA-approval drug lists and applied in clinical trial in different diseases (Table 1 and 2).

Antiestrogen resistant cell lines exhibit increased basal autophagy. Study had demonstrated combination of Tamoxifen or Faslodex (ICI) with hydroxychloroquine (HCQ) had different anti-estrogen responsiveness in vitro or in vivo which may affect by tumor microenvironment (i.e. chemokines, macrophage development/activity [12]. Inhibiting autophagy via autophagy-related genes (i.e. autophagyrelated gene (Atg) 5, Atg7, and p62/SQSTM1) silencing potentiated antiestrogen-mediated cell death, indicating that antiestrogen stimulated autophagy is prosurvival and a critical mechanism of therapy resistance [3]. Overall, it indicated that increased in autophagy activity in early recurring breast cancer when compared with breast cancer that never recurs. Moreover, elevated p62 is significantly correlated with poor survival in breast cancer patients [12], suggesting a role for autophagy in breast cancer reoccurrence. Manipulation of autophagy activity can be a potential therapy for chemotherapy in vitro or in vivo. Therefore, diagnosis or detection at an early stage is crucial to allow the application of treatments for increasing the life expectancy of the patient.

Table 1. Autophagy inhibitors during autophagy pathway. Modified from [13].

\begin{tabular}{|l|l|l|}
\hline Name & Mechanism & \\
\hline 3-Methyladenine & phosphoinositide3-kinase (PI3) inhibitor & $\begin{array}{l}\text { Autophagosome } \\
\text { formation }\end{array}$ \\
\hline Wortmannin & PI3-kinase inhibitor & $\begin{array}{l}\text { Autophagosome } \\
\text { formation }\end{array}$ \\
\hline LY294002 & PI3-kinase inhibitor & $\begin{array}{l}\text { Autophagosome } \\
\text { formation }\end{array}$ \\
\hline SBI-0206965 & Unc-51-like kinase 1 (ULK1) Inhibitor & $\begin{array}{l}\text { Autophagosome } \\
\text { formation }\end{array}$ \\
\hline Spautin-1 & $\begin{array}{l}\text { ubiquitin-specific peptidases (USP10) } \\
\text { and (USP13) inhibitor }\end{array}$ & $\begin{array}{l}\text { Autophagosome } \\
\text { formation }\end{array}$ \\
\hline SAR405 & $\begin{array}{l}\text { Vacuolar Protein Sorting Protein 18 and } \\
\text { 34 (Vps18 and Vps34) inhibitor }\end{array}$ & $\begin{array}{l}\text { Autophagosome } \\
\text { formation }\end{array}$ \\
\hline NSC185058 & $\begin{array}{l}\text { autophagy-related gene 4 (ATG4) } \\
\text { inhibitor }\end{array}$ & $\begin{array}{l}\text { Autophagosome } \\
\text { formation }\end{array}$ \\
\hline Verteporfin & Unknown & $\begin{array}{l}\text { Autophagosome } \\
\text { formation and } \\
\text { accumulation }\end{array}$ \\
\hline ROC325 & Unknown & Lysosome \\
\hline Lys05 & Unknown & Lysosome \\
\hline Chloroquine & Unknown & Lysosome \\
\hline Hydroxychloroquine & Unknown & Lysosome \\
\hline
\end{tabular}

Correspondence to: Li-Pin Kao, Department of Basic Medical Sciences, Purdue University, West Lafayette, Indiana, USA, E-mail: kaolipin@gmail.com

Key words: breast cancer, autophagy, autophagy-related inhibitors, chemotherapy

Received: February 03, 2018; Accepted: February 20, 2018; Published: February 23, 2018 
Table 2. Current Hydroxychloroquine (HCQ) clinical trials. Modified from [13].

\begin{tabular}{|c|c|c|c|}
\hline Treatment & Condition & Phase Trial & Reference \# at ClinicalTrials.gov \\
\hline $\mathrm{HCQ}+$ sunitinib malate & Adult solid neoplasm & I & NCT00813423 \\
\hline HCQ + vorinostat & Malignant solid tumor & I & NCT01023737 \\
\hline HCQ + sirolimus or vorinostat & Advanced cancers & I & NCT01266057 \\
\hline $\begin{array}{l}\text { HCQ + Protein kinase B (Akt) inhibitor } \\
\text { MK-2206 dihydrochloride (MK2206) }\end{array}$ & Advanced cancers & I & NCT01480154 \\
\hline HCQ as a single agent & Estrogen receptor positive breast cancer & $\mathbf{I}$ & NCT02414776 \\
\hline $\mathrm{HCQ}+$ gemcitabine & Advanced adenocarcinoma & I/II & NCT01506973 \\
\hline HCQ + Interleukin 2(IL-2) & Renal cell carcinoma & I/II & NCT01550367 \\
\hline $\mathrm{HCQ}+$ vorinostat & Colorectal cancer & I/II & NCT02316340 \\
\hline HCQ + gemcitabine/carboplatin & Small cell lung cancer & I/II & NCT02722369 \\
\hline HCQ + capecitabine & Pancreatic carcinoma & II & NCT01494155 \\
\hline HCQ as a single agent & Prostate cancer & II & NCT00726596 \\
\hline $\mathrm{HCQ}+$ Abraxane and gemcitabine & Pancreatic carcinoma & II & NCT01978184 \\
\hline
\end{tabular}

\section{Reference}

1. Garbar C (2017) Chemotherapy treatment induces an increase of autophagy in the luminal breast cancer cell MCF7, but not in the triple-negative MDA-MB231. Sci Rep 7: 7201.

2. O'Reilly EA, Gubbins L, Sharma S, Tully R, Guang MH, et al. (2015) The fate of chemoresistance in triple negative breast cancer (TNBC). BBA Clin 3: 257-275. [Crossref]

3. Cook KL, Shajahan AN, Wärri A, Jin L, Hilakivi-Clarke LA, et al. (2012) Glucoseregulated protein 78 controls cross-talk between apoptosis and autophagy to determine antiestrogen responsiveness. Cancer Res 72: 3337-49. [Crossref]

4. Zhang P, Liu X, Li H, Chen Z, Yao X, et al. (2017) TRPC5-induced autophagy promotes drug resistance in breast carcinoma via CaMKKbeta/AMPKalpha/mTOR pathway. Sci Rep 7: 3158. [Crossref]

5. 5Clarke R, Cook KL, Hu R, Facey CO, Tavassoly I, et al. (2012) Endoplasmic reticulum stress, the unfolded protein response, autophagy, and the integrated regulation of breast cancer cell fate. Cancer Res 72: 1321-31. [Crossref]

6. Thomas S, Thurn KT, Biçaku E, Marchion DC, Münster PN (2011) Addition of a histone deacetylase inhibitor redirects tamoxifen-treated breast cancer cells into apoptosis, which is opposed by the induction of autophagy. Breast Cancer Res Treat 130: 437-47. [Crossref]

7. Vazquez-Martin A, Oliveras-Ferraros C, Menendez JA (2009) Menendez, Autophagy facilitates the development of breast cancer resistance to the anti-HER2 monoclonal antibody trastuzumab. PLoS One 4: e6251. [Crossref]

8. Cook KL, Soto-Pantoja DR, Abu-Asab M, Clarke PA, Roberts DD, et al. (2014) Mitochondria directly donate their membrane to form autophagosomes during a novel mechanism of parkin-associated mitophagy. Cell Biosci 4: 16. [Crossref]

9. Clarke R, Shajahan AN, Riggins RB, Cho Y, Crawford A, et al. (2009) Gene network signaling in hormone responsiveness modifies apoptosis and autophagy in breast cancer cells. J Steroid Biochem Mol Biol 114: 8-20. [Crossref]

10. Schoenlein PV, Periyasamy-Thandavan S, Samaddar JS, Jackson WH, Barrett JT (2009) Autophagy facilitates the progression of ERalpha-positive breast cancer cells to antiestrogen resistance. Autophagy 5: 400-3. [Crossref]

11. Samaddar JS, Gaddy VT, Duplantier J, Thandavan SP, Shah M, et al. (2008) A role for macroautophagy in protection against 4-hydroxytamoxifen-induced cell death and the development of antiestrogen resistance. Mol Cancer Ther 7: 2977-87. [Crossref]

12. Cook KL, Wärri A, Soto-Pantoja DR, Clarke PA, Cruz MI, et al. (2014) Hydroxychloroquine inhibits autophagy to potentiate antiestrogen responsiveness in ER+ breast cancer. Clin Cancer Res 20(12): 3222-32. [Crossref]

13. Chude CI, RK (2017) Amaravadi, Targeting Autophagy in Cancer: Update on Clinical Trials and Novel Inhibitors. Int J Mol Sci. [Crossref]

Copyright: (C2018 Li-Pin Kao. This is an open-access article distributed under the terms of the Creative Commons Attribution License, which permits unrestricted use, distribution, and reproduction in any medium, provided the original author and source are credited. 